\title{
INFLUENCE OF PREBIOTICS ON THE BIOLOGICAL ACTIVITY OF LACTIC ACID BACTERIA
}

\author{
N. O. Kravchenko, O. M. Dmytruk, L. V. Bozhok, V. O. Aheyev, O. V. Holovach
}

Prebiotics are substrates that stimulate the growth and activity of the representatives of normocenosis of gastrointestinal tract of the macroorganism. This is a large group of substances that normally come to animals and birds as part of the diet. They are not either digested or absorbed in the stomach and small intestine; getting into the thick intestine they act as a nutrition medium for normal microflora [1]. The results of studies as for the influence of substances of prebiotic effect on bifido- and lactobacteria of human gastrointestinal tract (GIT) are sufficiently described in the literature [2-5]. However, the results as for the effects of these substances on the growth and activity of lactic acid bacteria (LAB) in the animals GIT are not covered enough. The study of this question will justify the scientific principles of new symbiotic preparations for animals that will contribute to the intensification of Livestock sector and the development of national biotechnology.

Materials and methods. 9 strains of lactic acid bacteria of Lactobacillus genus: L23, L-24, L-2/1, L-4P, L-51, L-16, L-61, L19, L-20, isolated from the GIT of calves aged up to 3 months, were used in the experiments.

Lactose in the form of Duphalac containing $66.7 \%$ of lactulose, chicory containing inulin up to $47 \%$ and metabolites of probiotic aerobic bacilli (MAB) of Bacillus subtilis species, obtained by autoclaving $\left(134^{\circ} \mathrm{C}, 60 \mathrm{~min}\right.$.) of daily culture, were used as prebiotics.

Six variants of media were prepared for the study to which the above prebiotics in various concentrations were included. Basal medium (BM) was used as control, which includes corn extract (1.5\%), whey $(20 \%)$, hydrolyzed milk $(15 \%)$, sodium citrate $(2 \%)$, manganese sulfate (about $0.02 \%$ ), $\mathrm{pH} 5.5$ (Table 1).
Table 1. Variants of media with
prebiotics

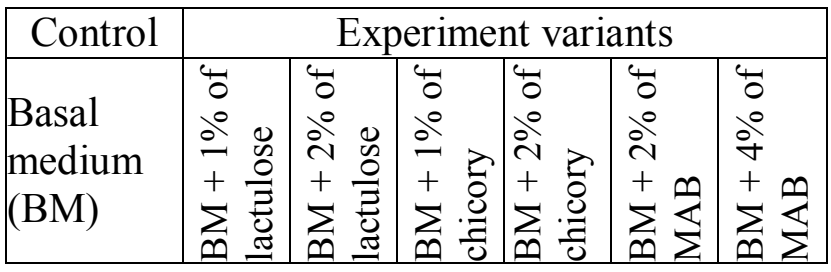

The investigated strains of $\mathrm{LAB}$ were cultured on the above media at the temperature $37^{\circ} \mathrm{C}$ for 5 days. Inoculums dose was $1.0 \pm 0.1 \%$. The concentration of viable cells was determined by 10 -fold dilution method [6]. The effect of prebiotics on LAB growth activity was assessed by stimulation index defined by the formula:

$$
\mathrm{SI}_{\text {g.a. }}=\frac{\mathrm{C}_{\mathrm{pr}}}{\mathrm{C}_{\mathrm{BM}}},
$$

where $\mathrm{SI}_{\text {g.a. }}$ - stimulation index of growth activity;

$\mathrm{C}_{\mathrm{pr}}$ - - concentration of bacteria in the medium with prebiotic;

$\mathrm{C}_{\mathrm{BM}}$ - concentration of bacteria in basal medium.

Proteolytic activity (caseic) of investigated strains of LAB was determined by casein hydrolysis area around colonies raised on milk agar [7]. The effect of prebiotics on $\mathrm{LAB}$ proteolytic activity was assessed by stimulation index defined by the formula:

$$
\mathrm{SI}_{\text {p.a. }}=\frac{\mathrm{HA}_{\mathrm{pr}}}{\mathrm{HA}_{\mathrm{BM}}}
$$

where $\mathrm{SI}_{\text {p.a. }}-$ stimulation index of proteolytic activity;

$\mathrm{HA}_{\mathrm{pr}}$ - hydrolysis area around LAB colonies grown in the medium with prebiotic;

$\mathrm{HA}_{\mathrm{BM}}$ - hydrolysis area around LAB colonies grown in basal medium. 
Antagonistic activity of investigated strains of $\mathrm{LAB}$ was determined by agar diffusion method [8]. Conditionally pathogenic microorganisms Salmonella typhimurium strain No 89 and Escherichia coli strain 055:K59 No 3912 were used as test-cultures. Record of the results was carried out by measuring the inhibition zone of testcultures. The effect of prebiotics on LAB antagonistic activity was assessed by stimulation index defined by the formula:

$$
\mathrm{SI}_{\text {a.a. }}=\frac{\mathrm{IZ}_{\mathrm{pr}}}{\mathrm{IZ}_{\mathrm{BM}}},
$$

where $\mathrm{SI}_{\text {a.a. }} \quad-$ stimulation index of antagonistic activity;

$\mathrm{IZ}_{\mathrm{pr}}$ - inhibition zone of test-cultures around $\mathrm{LAB}$ colonies grown in the medium with prebiotic;

$\mathrm{IZ}_{\mathrm{BM}}$ - inhibition zone of test-cultures around $\mathrm{LAB}$ colonies grown in basal medium.
Thus, the dependence of growth activity of the representatives of normoflora of gastrointestinal tract of young stock from the availability of lactulose in nutrient medium was determined in vitro. It is shown that the average index of growth stimulation of lactic acid bacteria in the medium with lactulose on the day 5 of cultivation exceeds 1.7 times the same indicator in the media with other prebiotics.

The stimulation of antagonistic activity of the representatives of normoflora of the digestive tract of young stock depending on the availability of prebiotics was established. The biggest stimulation of antagonistic activity of lactic acid bacteria to $S$. typhimurium and E. coli was found in the activity of metabolites of aerobic bacilli. The obtained results make a scientific basis for creating a symbiotic preparation for the livestock. 